\title{
Aspects géotechniques de l'accident du nouveau port de Nice
}

P. HABIB

Laboratoire

de Mécanique

des Solides

École polytechnique, 91128 Palaiseau.

Le glissement sous-marin qui s'est produit le 16 octobre 1979 lors de la construction du nouveau port de Nice a fait l'objet d'un très grand nombre de travaux pour tenter de déterminer les causes de l'accident. Ne disposant que de témoignages partiels et le corps du glissement ayant disparu, l'interprétation est très fragile. Après un rappel des faits et une description géotechnique du talus d'alluvions du delta du Var et des dépôts sur lesquels les pistes d'envol de l'aéroport de Nice ont été construites, différents scénarios sont décrits qui peuvent expliquer comment les alluvions se sont liquéfiées et ont engendré un glissement sans rapport avec l'exécution des travaux, glissement d'au moins huit millions de mètres cubes comprenant les terrassements faits pour la digue du nouveau port ainsí que le site même de la digue. Enfin, on évoque le risque sismique des talus sous-marins.

\section{Geotechnical aspects of the Nice} new harbour accident

The submarine slope failure which occured on Oct. 16th 1979 during the construction of the Nice new habour was extensively studied to try to find the origin of the accident. There is only incomplete testimonies and the main part of the slipped mass having disappeared, the interpretation is flimsy. After a geotechnical description of the alluvional slope of the Var delta and of the soils on which the Nice airfield was built, different scenarios are given to try to describe and explain how alluvions liquefied and created a slip having no relation with the harbour making, a slip of about eight millions cubic meters, including the fills of the new harbour dike and the soils of the very dike site. Finally seismic risk of submarine slopes are recalled.
} 


\section{Introduction}

Le 16 octobre 1979, il faisait à Nice un très mauvais temps avec du froid, du vent, des averses et une très mauvaise visibilité sur la mer. De sorte qu'il y avait très peu de gens sur les plages et sur la côte et, par conséquent, il n'y a eu qu'un petit nombre d'observateurs pour rapporter une vue même partielle du grave accident qui s'est produit au nouveau port, avec seulement une dizaine de témoins environ, répartis le long de la baie des Anges. Cet accident a causé des pertes en vies humaines ; il a été provoqué par un glíssement sousmarin, qui a engendré d'une part un phénomène de raz de marée tout le long du littoral autour de Nice dont les effets ont été ressentis au loin, et d'autre part la disparition d'une grande partie des travaux de remblaiement du nouveau port. A La Salis, à l'ouest, près d'Antibes, il $\mathrm{y}$ a eu notamment trois vagues destructrices, dont la première d'ailleurs n'a pas été la plus forte, avec, entre chacune, un retrait de la mer découvrant la plage jusqu'à trois mètres de profondeur. Par beau temps, on aurait sans doute pu avoir davantage de témoignages et on aurait été capable d'une vue d'ensemble du phénomène, mais il y aurait eu sans doute beaucoup plus de victimes.

Pour saisir l'origine de l'accident et analyser les phénomènes observés, des études très nombreuses et très complètes ont été entreprises. Elles ont été demandées d'une part, par la Mission d'Inspection Pluridisciplinaire (MIP), c'est-à-dire la commission administrative nommée par le gouvernement français, et d'autre part par la Direction Départementale de l'Équipement des AlpesMaritimes (DDE), maitre d'œuvre des travaux du port et de l'aéroport. Les avis des meilleurs experts du moment ont été recueillis et de nombreux organismes ont participé aux enquêtes : la gendarmerie pour les témoignages, la météorologie nationale et le CNEXO pour l'état de la mer, le BRGM, le LCPC, I'Université de Califormie (Berkeley) pour la géologie et la géotechnique, le LDG pour la veille sismologique, le tout avec des essais de laboratoire, des reconnaissances géotechniques sur le terrain, des reconnaissances sous-marines, etc. Il y a eu aussi, bien entendu, des analyses faites par les experts judiciaires. Pourtant, après tous ces travaux, mais du fait de l'absence d'une vision complète et globale de l'accident, il est extrêmement difficile de décrire aujourd'hui l'enchaînement des phénomènes de glissement et de raz de marée.

Dès le commencement des expertises, deux thèses ont été avancées; pour les uns, l'origine du raz de marée était le glissement qui a affecté le terre-plein sud du nouveau port, la vague que ce phénomène aurait engendrée étant responsable des dégâts sur la côte. Pour les autres, l'origine de l'accident était à rechercher dans un effondrement sous-marin, accident qui aurait engendré une première vague dont l'effet fut la destruction du nouveau port et le raz de marée sur la côte.

Au lendemain de l'accident, le journal Le Monde, du 18 octobre 1979, titrait : «Deux hypothèses pour la catastrophe de la Côte-d'Azur : avalanche sous-marine ou glissement de terrain ? x Bref, est-ce la poule qui a fait I'œuf ou l'œuf qui a fait la poule? Il n'est pas certain que l'on puisse apporter aujourd'hui la preuve formelle qu'une de ces deux interprétations soit fausse; on peut cependant tenter de proposer des modèles d'accident qui intègrent bien les observations techniques faites.
Rappelons enfin que les aspects géologiques de cet accident ont fait l'objet d'une présentation orale de J. Goguel devant le Comité Français de Géologie de l'Ingénieur et que le but du présent article est de préciser certains aspects géotechniques du ou des glissements de terrain du 16 octobre 1979.

\section{Généralités sur les grands glissements de terrain}

Bien que les glissements de terrain soient des accidents relativement courants, nos connaissances sur la genèse des grands glissements, c'est-à-dire supérieurs à dix millions de mètres cubes, sont très limitées. Il y a très peu de bonnes descriptions de grands glissements, parfois parce que les observateurs compétents ont été emportés par le désastre (Vaïont [4]), le plus souvent parce qu'il n'y en avait pas ou que ceux qui étaient présents ont eu rapidement d'autres occupations que de prendre des notes. Le film du Norwegian Geotechnical Institute d'Oslo sur le grand glissement de Rissa, en Norvège, est, à ce titre, tout à fait exceptionnel puisqu'il a permis de saisir pratiquement toutes les phases d'un accident.

En fait, on ne sait même pas s'il y a une systématique des grands glissements. Compte tenu des masses mises en jeu, des distances concernées, il est invraisemblable que les mouvements puissent se produire simultanément, et peu crédible qu'ils commencent par le haut : il semble que les très grands glissements de sol soient plutôt régressifs, c'est-à-dire qu'ils commencent par une ou par plusieurs ruptures à partir du bas et se développent ensuite progressivement vers le haut. Le mécanisme des avalanches semble inverse : l'avalanche commence à partir du haut de la pente et s'écroule en s'amplifiant vers le bas. Mais l'avalanche est plus un accident des pentes neigeuses ou rocheuses que des talus de terre et sa vitesse est généralement plus grande que celle des glissements de terrain. Les vitesses de ces derniers sont généralement faibles, voire très faibles en dehors des crises paroxysmiques, où elles atteignent quelques kilomètres à l'heure sauf dans les cas de liquéfaction des loess, des argiles sensibles ou des sables lâches où de véritables fleuves de boue peuvent s'écouler comme des liquides. Signalons cependant le glissement de Vaïont $\left(300.10^{6} \mathrm{~m}^{3}\right)$, déjà cité, qui a largement dépassé la vitesse de $100 \mathrm{~km} / \mathrm{h}$ sans liquéfaction (Fig. 1), avec une plaque quasi intacte ou, tout au moins, sans grave désorganisation de sa structure géologique [1]. A Rissa $\left(10.10^{6} \mathrm{~m}^{3}\right)$ des plaques superficielles rigides ont flotté sur un fleuve de boue, entraînées à près de $30 \mathrm{~km} / \mathrm{h}$. Le glissement de Tuve [3], en Suède $\left(6.10^{6} \mathrm{~m}^{3}\right)$ intéressant une grande partie d'argile sensible a été lui aussi régressif, comme celui de Rissa.

Les glissements sous-marins sont encore plus mal connus : on ne dispose pratiquement d'aucune observation et on ne les connait que par leurs effets. Des glissements sous-marins de plusieurs centaines de millions de mètres cubes se sont produits et ont été identifiés. Des observations ont montré que des glissements sousmarins se sont produits un mois après l'action d'un agent déclenchant dûment identifié. Comme pour les glissements à terre, le grand glissement sous-marin monolithique de terre paraît exclu et le caractère régressif parait probable. Mais, en revanche, le mécanisme 


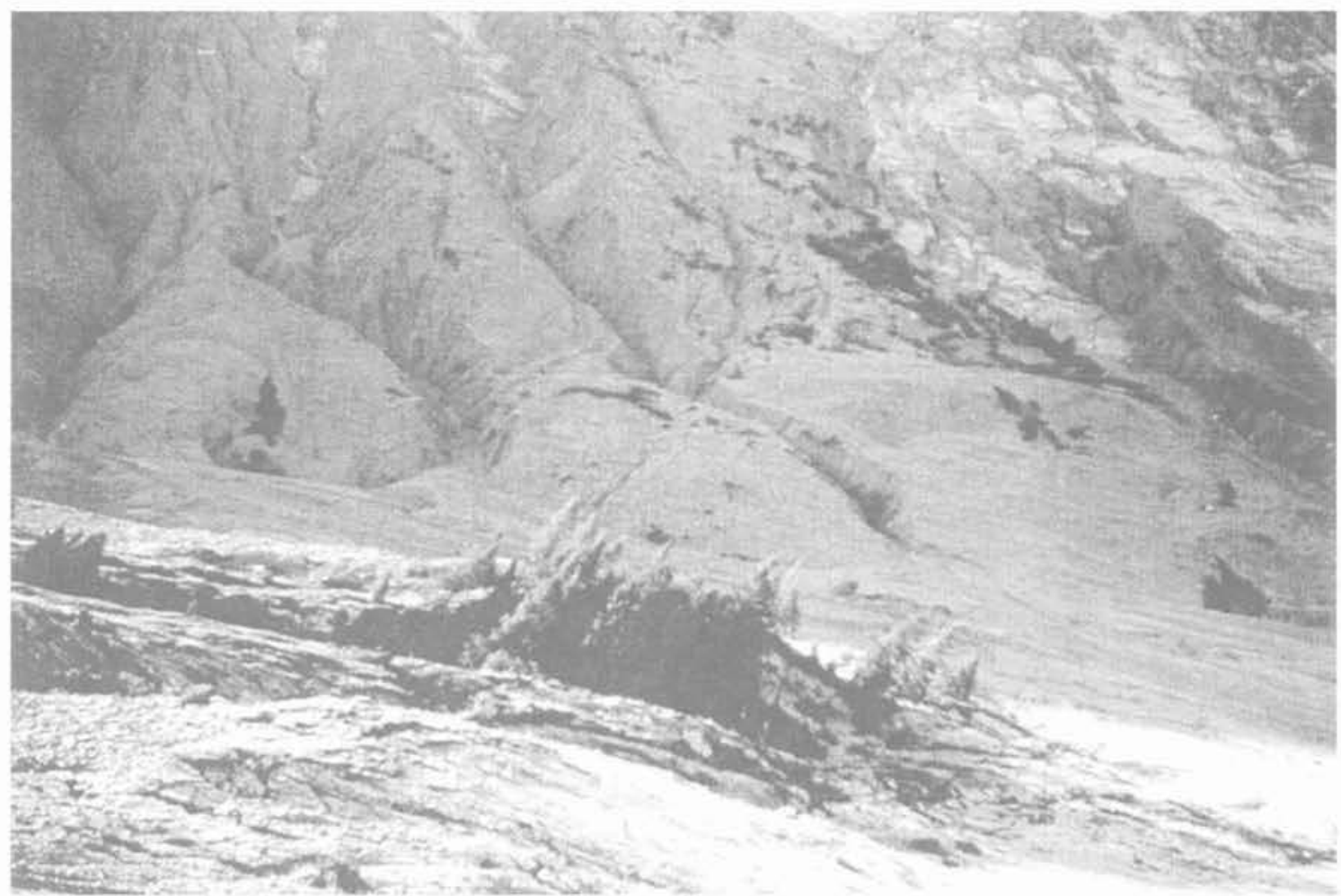

FIG.1 Vaïont : glissement du mont Toc. Il est assez étonnant de penser que ce boqueteau boisé a glissé à une vitesse de $100 \mathrm{~km} / \mathrm{h}$.

Vaiont : mount Toc slide. It is surprising to think this small wood had slipped with a $100 \mathrm{~km} / \mathrm{h}$ speed.

d'avalanche est certainement possible. Dans un glissement à terre, ce qui provoque l'arrêt du glissement, c'est essentiellement le changement de géométrie : le bourrelet qui se produit au pied du glissement diminue le moment moteur et augmente la résistance au mouvement par son poids et par son frottement. Dans l'eau, le poids moteur est diminué mais le poids du bourrelet est aussi moins efficace, puisqu'il est réduit par la poussée d'Archimède, et le frottement peut disparaître, annulé par la lubrification de l'eau : un mouvement accéléré devient possible, et on revient au phénomène d'avalanche. Il n'est pas douteux que les nombreux canyons à fond plat qui existent autour du plateau du delta du Var, dont celui dit du Paillon, sont des couloirs d'avalanches par où doivent passer, de temps à autre, des chasses de sédiments sous forme de courants de densité qui assurent au talus du delta du Var un équilibre dynamique au rythme lent des phénomènes géologiques. Seul le mécanisme de l'avalanche hydrodynamique permet d'expliquer les distances atteintes par les débris et les vitesses du ou des courants de densité lors de l'accident du 16 octobre 1979.

Il est hautement probable que dans le glissement. qui a entraîné une partie du nouveau port, les deux mécanismes, régression et avalanche, se sont produits, et la zone d'initiation du glissement qui a emporté le nouveau port se situe probablement au sein de la partie la plus raide du talus du plateau du Var. Il paraît hautement probable que des mécanismes régressifs avec des liquéfactions se sont produits au-dessus de ce point critique et vers la digue du nouveau port. Il est hautement probable qu'une avalanche s'est produite dans la partie située en dessous du point critique. Il faut dire aussi que la stabilité du talus du delta du Var était précaire avant l'accident.

\section{3}

\section{Le talus du delta du Var}

Sa stabilité était précaire, comme celle de tous les talus pourrait-on dire. Pour être plus explicite, on peut dire que tout talus à un moment donné a été instable et un talus en formation est, presque par définition, à la limite de l'équilibre.

Le talus du delta du Var est une formation quaternaire. Les sables denses de la base sont encapsulés par les silts sableux, qui sont eux-mêmes surmontés par des argiles silteuses. La description de ces derniers matériaux correspond à des silts, des argiles silteuses ou des silts sableux. Le cceur du talus contient une nappe d'eau douce en surpression de $5 \mathrm{~m}$ par rapport au niveau de la mer. Les formations silteuses sont souvent laminées: des passages de sables lâches y existent, avec de très faibles densités, jusqu'à $15 \%$ ou $20 \%$ en densité relative. Une coupe approximative est donnée sur la figure 2.

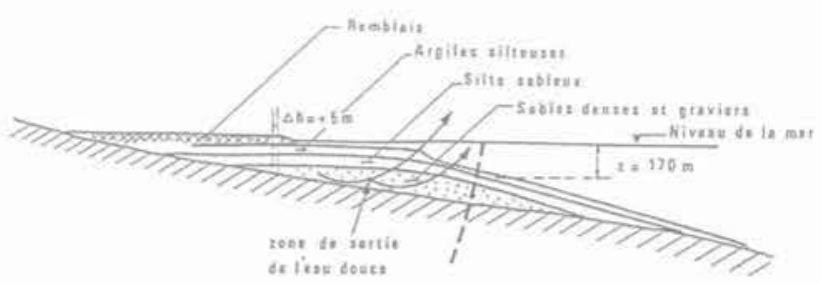

FG. 2 Coupe schématique du talus du delta du Var au $1 / 25000^{\mathrm{e}}$.

Schematic vertical section of Var delta slope $\left(1 / 25000^{\mathrm{c}}\right)$. 
La partie en pente très douce, presque un plateau, auprès de la côte, est celle qui a été gagnée sur la mer pour constituer le complexe aéroportuaire avec les pistes de roulement et les deux grandes bandes d'envol, Le remblaiement du plateau du delta a commencé dès 1945 , d'abord avec des galets du Var puis, devant la menace d'épuisement du gisement, avec des remblais rocheux provenant des grands travaux autoroutiers, ou autres, de la région niçoise ; au début on a remblayé pour les pistes par $10 \mathrm{~m}$ d'eau; pour les travaux du nouveau port, le remblaiement était fait par $15 \mathrm{~m}$ d'eau d'abord par clapage puis par déversement pour les couches supérieures. Le bord supérieur du remblaiement était à $150 \mathrm{~m}$ du bord du talus dont la pente près du bord était de l'ordre de 27 à $31^{\circ}$ et plus bas, moyenne pour tout le talus, de l'ordre de $20 \%$ (soit $12^{\circ}$ ) jusqu'à $1500 \mathrm{~m}$ de profondeur.

Du fait de la différence des densités de l'eau de mer et de l'eau douce, la surpression de $5 \mathrm{~m}$ de l'eau de la nappe des sables à la base du talus du delta du Var peut correspondre à une communication directe entre la mer et les graviers et les sables à une profondeur de 5 $\frac{5}{1,03-1,00}=170 \mathrm{~m}$. Mais ceci est peu vraisemblale. En

effet, si le système est clos, au-dessus de - $170 \mathrm{~m}$ le gradient hydraulique est positif dans la couche d'argile et l'eau douce sort du talus. A la cote $-170 \mathrm{~m}$, le gradient hydraulique est nul. En-dessous de $-170 \mathrm{~m}$, le gradient serait négatif et l'eau de la mer pourrait pénétrer dans le talus. Depuis le temps que cette situation existe, un équilibre a dû s'établir avec un horizon d'eau salée dans le talus du delta à la cote $-170 \mathrm{~m}$, de l'eau douce au-dessus et probablement une zone de transition.

On peut tenter un calcul approximatif du débit d'eau douce qui s'échappe du talus du delta du Var. Avec une perméabilité des argiles de l'ordre de $10^{-8} \mathrm{~m} / \mathrm{s}$, et en admettant que la couche externe soit peu épaisse, par exemple de l'ordre de $5 \mathrm{~m}$, soit un gradient moyen $\mathrm{j}=1 / 2$ (c'est-à-dire 0 à la cote $-170 \mathrm{~m}$ et 1 à la cote 0 ). Le débit des infiltrations serait de :

$$
\mathrm{Q}=\mathrm{K} \cdot \mathrm{i} . \mathrm{S}=10^{-8} \times \frac{1}{2} \times 1000 \mathrm{~m} \times 1 \mathrm{~m}=5 \mathrm{~cm}^{3} / \mathrm{s} \text { par mètre de côte, }
$$

ce qui est insignifiant par rapport à l'alimentation naturelle des sables sous-jacents. Si la perméabilité des sables est de l'ordre de $10^{-4} \mathrm{~m} / \mathrm{s}$ on en déduit que le gradient d'alimentation des sables est de quelques millièmes, ce qui est très petit et très raisonnable. Même si ces calculs doivent être considérés comme de grossières approximations, il apparaît que le débit de percolation de l'eau douce est très petit.

La configuration hydraulique souterraine rare du talus du delta du Var mérite d'être rapprochée de la forme lobée de ce talus, qui est tout à fait caractéristique de la topographie de cette formation (Fig. 3). Cette correspondance n'a pas fait l'objet d'une étude systématique, mais il est classique que l'émergence d'une nappe à partir d'un talus à l'air libre est une situation instable qui peut donner naissance au phénomène de renard : il suffit d'une faiblesse mécanique locale du talus pour que la terre soit entraînée par le courant d'eau ; les lignes de courant aboutissant en ce point deviennent alors un peu plus courtes, le gradient hydraulique et les forces de courant y sont alors un peu plus grands; le point correspondant devient plus instable, le phénomène peut alors s'amplifier.
On peut imaginer un mécanisme analogue sur le talus du delta du Var. Les alluvions se déposent régulièrement sur l'ensemble de la pente. Sur les crêtes des lobes, le gradient hydraulique est plus faible que dans les thalwegs oủ la couche argilo-silteuse en place est moins épaisse. Les dépôts en fond de thalweg se consolident donc moins bien que ceux des crêtes; on notera que ce fait est indépendant du débit à travers la couche argileuse, mais ne dépend que des contrastes de perméabilité, les couches les moins perméables étant les plus sensibles à ce phénomène. Les thalwegs apparaissent alors comme des lieux privilégiés de déclenchement des décharges d'alluvions instables qui se produisent périodiquement et dont la trace est marquée par le réseau de chenaux à fond plat (appelés improprement canyons), qui enserrent le pourtour du talus du delta du Var, dont celui du Paillon, qui en fait appartient clairement à ce système d'effondrement des déjections alluviales.

Il est difficile d'échapper à une telle interprétation : un talus lobé ne peut se former que si les lobes s'agrandissent ou si les creux s'approfondissent. Or, imaginer que les alluvions se déposent spécialement sur les crêtes est incompréhensible : les alluvions doivent se déposer d'une façon homogène et probablement plus abondamment dans les sillons. Ceux-ci doivent alors se vider périodiquement pour subsister. Un mécanisme de chasse à partir des sillons, par exemple à partir du sillon de l'accident du 16 octobre 1979, parait donc hautement probable.

Il résulte de ces considérations que l'on ne peut pas connaître l'âge des matériaux qui sont partis, ni leurs propriétés mécaniques. Les datations au Carbone 14, faites à différentes profondeurs dans le talus, ont indiqué les âges suivants pour les matériaux du cône d'alluvions :

$$
\begin{aligned}
& \text { - à } 32,7^{m} \text { : } \quad 6750 \text { ans BP (1), } \\
& \text {-à } 67,5 \mathrm{~m} \text {; } 11460 \text { ans BP, } \\
& \text { - à 69,5 m : } 12760 \text { ans BP. }
\end{aligned}
$$

Il est probable, et même à peu près certain, que les matériaux qui ont disparu dans le glissement du 16 octobre 1979 étaient beaucoup plus récents (c'est-à-dire quelques miliers, voire quelques centaines d'années). Du même coup, les comparaisons de stabilité faites à partir des caractéristiques mécaniques mesurées sur le même talus, mais ailleurs, ne sont pas valables. Elles donnent des bornes supérieures, car il est bien évident que ce qui est parti devait être moins résistant que ce qui est resté. Par exemple, ce qui est resté sur la rive gauche de l'effondrement et qui présente un talus très raide dont la pente est voisine de $45^{\circ}$ ne serait pas stable si l'on faisait un calcul de stabilité avec les caractéristiques mécaniques des argiles silteuses. Ceci démontre que les matériaux de crêtes (rive gauche de l'effondrement) sont bien mieux consolidés que ceux qui sont partis.

(1) Dans la notation BP (Before Present), I'origine du temps présent est 1950 . 


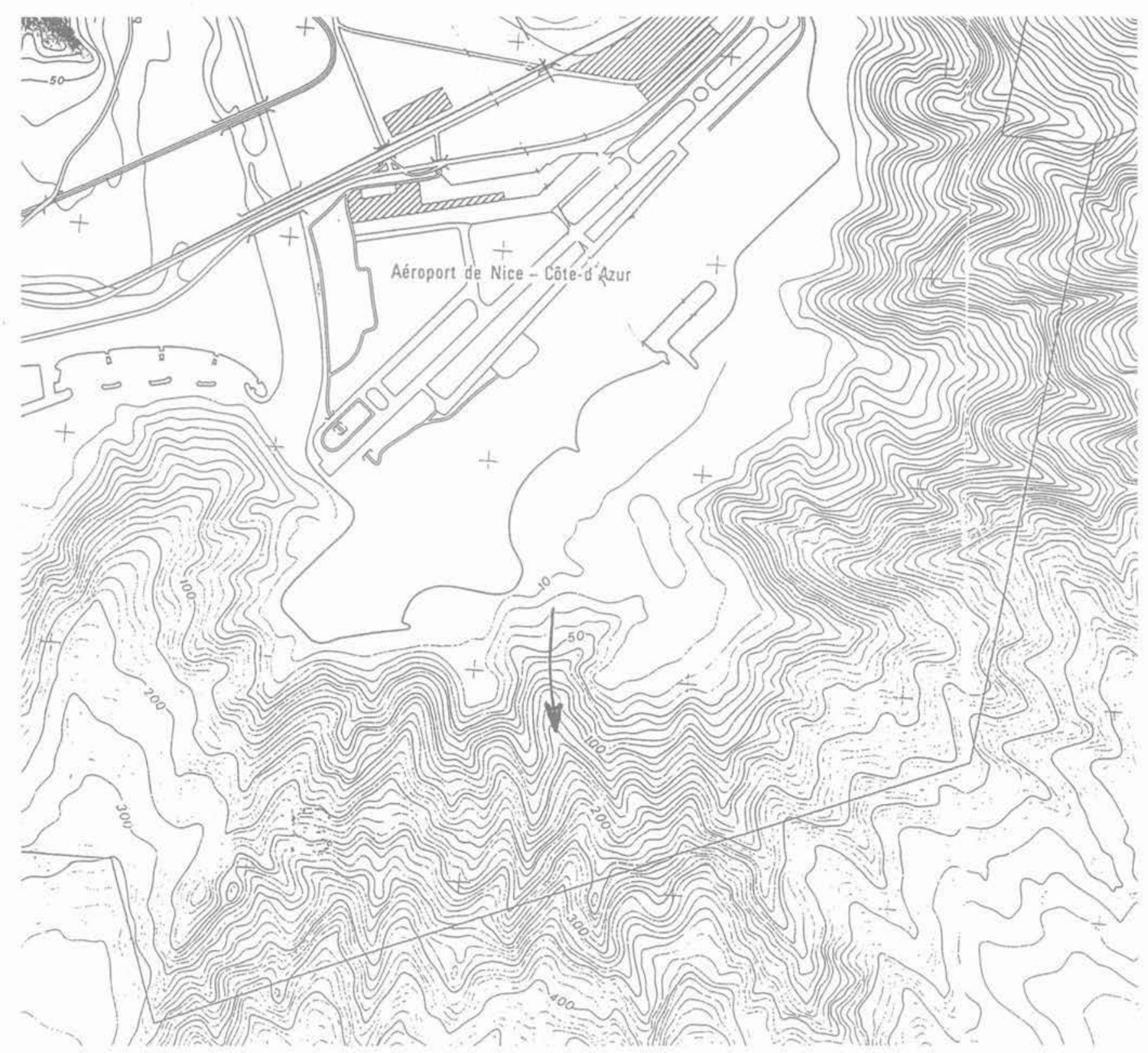

FIG.3 Topographie du fond de la mer après l'accident. Le canyon du Var est parfaitement visible avec son fond plat. Mais sur le talus, on distingue de très nombreux sillons dont la dírection est voisine des lignes générales de pente du talus. Le début de la trajectoire de l'effondrement du 16 octobre 1979 est marqué par une flèche.

Sea bed topography after the slide. The biginning of the october 16 th, 1979 slide trajectory is shown with an arrow.

\section{3}

\section{Les données matérielles et les événements}

\section{1}

\section{Chronologie des événements sur la côte}

C'est une banalité de répéter après tous les historiens qu'il est extrêmement difficile d'apprécier la valeur d'un témoignage séparé de son contexte, même lorsqu'il est écrit et enregistré. Il faut cependant entrer un peu dans le détail de la chronologie des événements. Dans le cas de l'accident de Nice, une circonstance exceptionnelle a permis de connaitre l'heure de la chute du nouveau port avec une extrême précision : les matérieis qui s'effondraient ont été vus par les personnels de la tour de contrôle de l'aéroport de Nice qui ont pu ainsi noter l'instant exact de cette partie de l'accident avec une rigueur professionnelle; la chute de la première grue a été notée à $13 \mathrm{~h} 57 \mathrm{~min} 30 \mathrm{~s}$ et celle de la deuxième grue à 14 h 01 min $30 \mathrm{~s}$, ce qui représente une durée d'environ 4 min à 5 min pour la disparition de la digue du nouveau port. Compte tenu de l'hésitation dans le début d'une chute, on peut affirmer que ces horaires sont connus à 15 secondes près. Pour le reste des événements, on dispose d'une dizaine de témoignages d'observateurs divers dont la relation avec le temps est beau- 
coup moins affirmée. Ils ont donné le long de la baie des Anges, et sur la côte, des informations certes très exactes, mais avec des imprécisions sur l'heure d'apparition des phénomènes, de l'ordre de \pm 2 min à \pm 3 min, ce qui est déjà très remarquable mais insuffisant dans le cas présent, comme on le verra plus loin. Par ailleurs, on n'est pas toujours tout à fait certain que le tout début du phénomène ait été observé.

Il y a, en outre, les observations faites au port de Saint-Laurent-du-Var oủ l'adjoint de l'officier du port a lu un temps sur une pendule avec une précision de l'ordre de la minute et où l'officier du port a étalonné ensuite l'heure de cette pendule par rapport à l'heure officielle. S'il est certain que ce témoignage correspond au début du phénomène de retrait de la mer sur la côte à Saint-Laurent-du-Var, il n'est pas impossible, toutefois, que le moment où la baisse du plan d'eau a été effectivement perçue soit un peu en retard sur le début de l'événement. En effet, la vitesse de descente du plan d'eau a été de l'ordre de 1 à $2 \mathrm{~cm} / \mathrm{s}$ et cela peut passer inaperçu (1) pendant un certain temps. En revanche, quand une darse se vide, les tourbillons de l'eau, les mouvements des bateaux, etc. sont beaucoup plus sensibles. Ce témoignage situe le phénomène entre $13 \mathrm{~h}$ 56 min 30 s et 13 h 57 min 30 s. Le début du reflux à Saint-Laurent-du-Var a été situé par la MIP à $13 \mathrm{~h} 57 \mathrm{~min}$ 30 s alors que le professeur Seed estime qu'à $13 \mathrm{~h} 56 \mathrm{~min}$ 30 s le premier reflux était déjà en cours.

Ce mouvement était le précurseur d'un raz de marée qui allait mettre en résonance la cuvette formée par la baie des Anges et ses prolongements de crêtes sousmarines à l'est et à l'ouest, avec une période d'environ $8 \mathrm{~min}$ et des oscillations ressenties pendant plusieurs heures d'Antibes à Beaulieu, en passant par Saint-Laurent-du-Var et Villefranche (Fig. 4),

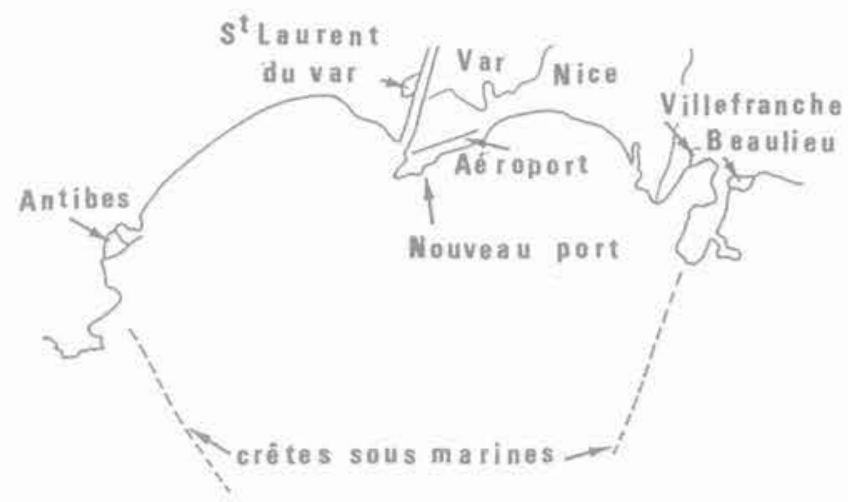

fig. 4 Plan de la zone intéressée par le sinistre du 16 octobre 1979.

Map of the places affected by the october 16 th. 1979 sinister.

Les autres témoignages dans les différents points de la côte ont une certaine imprécision sur l'heure; on ne sait pas toujours si c'est la même période qui est notée par chacun, ni si le début du phénomène est strictement le même partout (une montée ou une baisse de plan d'eau) et en se souvenant que la mise en résonance de n'importe quel système est précédée d'un régime transitoire au début du mouvement, avec d'abord une

(1) La même remarque vaut évidemment pour tous les témoignages où l'eau de la mer bordait un mur vertical. Sur une plage en pente douce, il n'en serait pas de même. pseudo-période qui, ici, a dû se coupler avec la période propre de la vague créée par l'íncident sous-marin. En fait, et notamment parce que les témoignages chronologiques étaient moins nombreux à l'est de l'aéroport qu'à l'ouest, il est même difficile d'affirmer, à partir de ces données, que les maximums de flux étaient en opposition des deux côtés de la baie des Anges. Mais ce mode d'oscillation est le seul qui puisse s'amortir en quelques heures : une oscillation pulsant en phase sur la côte aurait irradié toute son énergie vers le large et se serait amortie en quelques oscillations, ce qui manifestement n'a pas été le cas.

Dans ces conditions, il est pratiquement impossible de remonter, à partir des manifestations horaires sur la côte, vers la source et cela d'autant plus que la vitesse de la vague varie avec la profondeur h de la mer. La célérité de l'onde à la surface de l'eau est égale à $\sqrt{\text { gh }}$ lorsque h est constant, ce qui ici n'est jamais vérifié, le relief sous-marin de la baie des Anges étant même très tourmenté autour de l'aéroport de Nice. Il en résulte que les trajectoires des vagues ne sont pas rectilignes et qu'il n'est pas possible de retrouver la source de la perturbation.

Si le raz de marée qui s'est produit sur la côte est dû à un effondrement sous-marín, on peut se demander quel type de perturbation cela peut engendrer. A priori, l'effet principal en surface d'un effondrement sousmarin doit se trouver à la verticale de l'accident sous forme d'une dépression initiale du niveau de la mer qui, en irradiant un rond dans l'eau aux alentours, fait apparaitre d'abord un creux d'onde (Fig. 5). C'est bien ce qu'ont indiqué les trois marégraphes en service dans la baie des Anges ; d'ailleurs, cela a été bien constaté au port de Saint-Laurent-du-Var. Dans une station située environ à $1 \mathrm{~km}$ à l'est du nouveau port, la dépression première n'a pas été nettement perçue, et le premier signal véritablement enregistré a été une crête de vague vers 14 h 01. Cependant, on doit dire qu'il n'y avait pas d'observateur potentiel disponible à cet endroit au moment où un premier reflux se serait produit. Il n'est pas non plus évident qu'un premier reflux ait eu lieu en cette zone. Cette situation particulière montre la constante ambiguité des témoignages partiels et la difficulté d'obtenir des dèductions certaines.

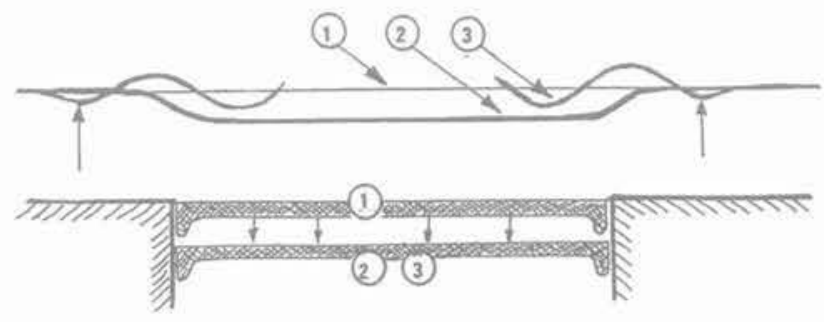

FG. 5 Si un piston s'enfonce dans un cylindre situé au fond de la mer en passant de la position (1) à la position (2), une dépression apparaît à la surface de l'eau initialement horizontale. Ultérieurement des oscillations (3) vont se produire, mais on voit que le précurseur du « rond dans l'eau » ainsi engendré est un creux d'onde. Sinking of a piston in the bottom of the sea. 
Le témoignage des marégraphes conforte donc le principe de la dépression initiale du plan d'eau après un effondrement sous-marin ; ce phénomène est probablement d'autant plus valable que l'accident sous-marin est profond.

Mais il est bien clair qu'un accident superficiel engendrerait simultanément et localement un petit gonflement initial du plan d'eau (Fig. 6). En effet, si l'effondrement sous-marin correspond à un affaissement du sol, il faut bien que le volume disparu réapparaisse quelque part et, par exemple, au pied du glissement où se situe habituellement le bourrelet [7]. Il peut donc y avoir frontalement, et dans la direction du glissement, un premier signal précurseur qui soit une crête de vague et non un creux, suivi évidemment ensuite de l'effet principal correspondant à la grande dépression. Le signal précurseur est plus petit que la dépression suivante, puisque son origine se situe plus profondément dans la mer que la tête de l'effondrement, et il est localisé vers la direction du mouvement général de l'écoulement solide. D'après la MIP un tel phénomène a probablement été observé à Antibes.

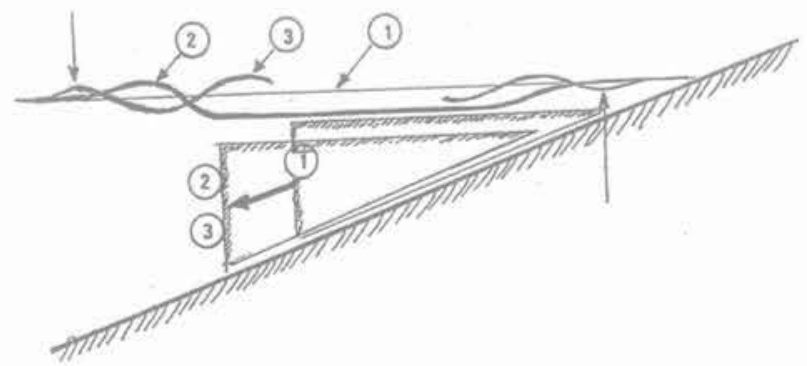

FIG.6 Si un bloc glisse au fond de la mer avec un mouvement de translation oblique qui le mène de la position (1) à la position (2), il se forme au-dessus de l'accident une dépression du niveau de la mer initialement horizontale. Mais, vers l'avant du glissement, on observe un gonflement du niveau de I'eau. Ultérieurement des oscillations (3) vont se produire mais cette fois-ci le précurseur vers l'avant du mouvement sera un sommet d'onde. Latéralement et à l'arrière, le précurseur sera évidemment un creux comme dans le cas de la figure 5 .

Sliding of a wedge along a slope in the bottorn of the sea.

Aucun séisme n'a été enregistré avant l'accident. Le réseau de veille sismique n'a rien détecté avant 13 h 57 , tout au plus une montée du bruit de fond à partir de $13 \mathrm{~h} 57$ ou de $13 \mathrm{~h} 58$, avec une agitation maximale très discrète entre 14 h $03-14$ h 04 et 14 h 07 - 14 h 08 , qui s'est atténuée à partir de $14 \mathrm{~h} 10$.

Cette agitation peut être attribuée aux entrechoquements des blocs de pierres du remblai en cours d'effondrement. Rappelons que vers $14 \mathrm{~h} 10$ les remblais du nouveau port avaient probablement effectué un trajet d'au moins 3 à $4 \mathrm{~km}$; rappelons aussi que la vitesse de propagation des ondes sonores dans le sol est comprise entre 2 et $5 \mathrm{~km} / \mathrm{s}$.

\section{Le glissement sous-marin près du nouveau port}

On ne dispose pas d'une bathymétrie complète du talus du delta du Var faite avant l'accident, et il y a une lacune entre $-100 \mathrm{~m}$ et $-500 \mathrm{~m}$. En extrapolant jusqu'à $-150 \mathrm{~m}$ ce qui était connu entre 0 et $-100 \mathrm{~m}$ et en faisant la différence avec la bathymétrie après l'accident (Fig. 7), il apparaît qu'environ huit millions de mètres cubes ont disparu, dont quatre millions (1) étaient constitués par l'emprise et par les remblais du nouveau port. Sur la figure 7 , l'isobathe $-20 \mathrm{~m}$ est indiquée en pointillé avant l'accident ainsi que la position de la digue destinée à la darse du nouveau port. Après l'accident, la profondeur à cet emplacement était de $-50 \mathrm{~m}$.

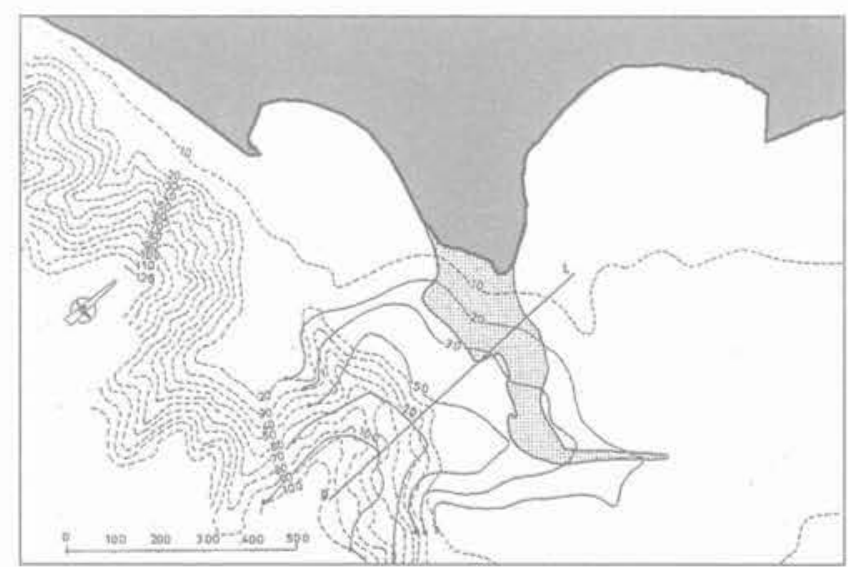

FIS.7 Plan des lieux avant et après le sinistre. Map of the new harbour before and after the sinister.

\section{2.}

\section{Hypothèse d'un grand glissement sous-marin} près du nouveau port

A partir de la rupture initiale, à 13 h 57 , un courant de densité s'est formé rejoignant le canyon sous-marin du Var et s'écoulant vers les grands fonds. Ce courant a rompu à $80 \mathrm{~km}$ de là le câble téléphonique GênesBarcelone, immergé à $-2520 \mathrm{~m}$, à 18 h 45 , puis 30 km plus loin le câble Gênes-Sassari, immergé à $-2600 \mathrm{~m}$, à 22 h 50 . La vitesse moyenne du courant de densité a donc été de l'ordre de $17 \mathrm{~km} / \mathrm{h}$ dans la première partie du trajet, et de l'ordre de $7 \mathrm{~km} / \mathrm{h}$ dans la seconde. Par une effet d'avalanche au cours de transport, le volume des sédiments entraînés a énormément grossi : l'estimation très imprécise de l'étendue et de l'épaisseur des nouveaux sédiments laisse penser qu'il est peut-être arrivé 100 millions de $\mathrm{m}^{3}$ de matériaux en fin de parcours.

(1) 2,5 millions de $\mathrm{m}^{3}$ pour les remblais d'après la MIP. 
Hypothèse d'un grand glissement sous-marin au large

Dans l'hypothèse où l'essentiel des matériaux arrivés en fin de parcours aurait été fourni par des glissements très importants au voisinage du confluent des canyons sous-marins du Var et du Paillon, la vitesse moyenne du courant de densité serait de l'ordre de $15 \mathrm{~km} / \mathrm{h}$ dans la première partie du trajet, entre le confluent des canyons et le premier câble et de $7 \mathrm{~km} / \mathrm{h}$ dans la seconde partie. estimations très voisines des précédentes ce qui ne permet pas d'utiliser ce critère pour trancher entre les deux hypothèses. Signalons cependant que la modélisation proposée par Mulder [6] estime la vitesse initiale à plus de $50 \mathrm{~m} / \mathrm{s}$.

Quelle que soit l'hypothèse envisagée, l'accident restera probablement un cas d'école de géologie, pour illustrer le transport à grande distance de matériaux par un courant de densité sous-marin, et le phénomène qui s'est produit à Nice le 16 octobre 1979 entre tout à fait dans la statistique portant sur 25 cas, donnée par Edgers et Karlsrud (1982) [2], tant pour le volume transporté que pour la distance couverte par les débris ou pour les variations de la vitesse au cours du mouvement.

\section{4 \\ La liquéfaction}

La rapidité de la disparition du nouveau port et la forme du talus sous-marin résiduel qui est en partie convexe et finalement avec une pente assez douce à l'emplacement de ce qui fut le nouveau port font penser que le sol des fondations s'est véritablement liquéfié. L'expression liquéfaction des sols est parfois employée avec différentes significations. Il importe d'être très clair sur ce sujet et de définir avec précision les différents concepts qui peuvent être sous-jacents. C'est ce qui va être tenté maintenant.

\section{1}

\section{Liquéfaction des sables fins saturés sous l'effet d'un chargement statique (B. Seed)}

Reprenant les études antérieures de Casagrande et Castro, Bolton Seed a fait un travail tout à fait original pour montrer que les sables lâches saturés pouvaient être placés dans un état mécanique métastable : il a travaillé sur le sable de la rivière Sacramento et sur le sable de Nice. Ce dernier est plus délicat à manier à cause de la présence de mica, qui rend la saturation des échantillons plus difficile et plus lente. Mais la propriété mise en évidence par B. Seed est une propriété extrêmement générale qui est valable pour tous les sables fins saturés dans un état très lấche, c'est-à-dire avec des densités relatives de l'ordre de $30 \%$ à $40 \%$.

Sous un état de contraintes triaxiales, tel que le rapport des contraintes principales soit de l'ordre de celui qu'on pouvait imaginer sur le talus du delta du Var, l'expérience montre qu'en condition de non-drainage (c'està-dire pour une sollicitation rapide), une très légère augmentation du déviateur des contraintes provoque un véritable phénomène de fluage, c'est-à-dire une déformation du matériau à vitesse constante. Ce phénomène, dans un délai plus ou moins long, s'accélère et aboutit à une perte complète de résistance par transfert des contraintes effectives vers la pression interstitelle. La structure du sable semble se désorganiser lentement vers une situation où les grains n'ont pratiquement plus de contact les uns avec les autres et flottent dans le liquide interstitiel. Le phénomène de fluage est capable de persister, même si, après avoir été déclenché par une petite augmentation de la contrainte déviatorique, on supprime cette petite augmentation pour revenir à l'état initial. C'est ceci qui justifie le mot métastable employé plus haut. Le phénomène de fluage est arrêté si l'on permet le drainage de l'échantillon. C'est-à-dire que le phénomène est régi par la vitesse de contrainte avant que soit atteint le maximum de la résistance, puis par la vitesse de déformation après ce maximum. Une augmentation de la pression interstitielle à partir de l'état initial ne provoque pas la mise en liquéfaction du sable, tout au moins pour des incréments raisonnables de la pression des pores ; bien entendu, comme pour tous les sables même à forte densité, une augmentation forte de la pression interstitielle peut engendrer la rupture du matériau en diminuant également les contraintes effectives, mais une petite augmentation de la pression interstitielle peut passer inaperçue.

Les difficultés expérimentales pour mettre en évidence ces propriétés sont grandes cornme pour toute étude d'un phénomène métastable. La principale complication réside dans le fait que les échantillons de sables lâches se compactent spontanément au moindre choc, à la moindre vibration; la saturation de ces matériaux est particulièrement délicate ; à titre indicatif, la préparation d'un échantillon peut durer huit heures et le contrôle de la saturation est évidemment une partie importante et délicate de l'essai.

Dans des conditions de chargement drainé (c'est-àdire lent dans la nature), de telles structures granulaires sont stables et ne se tassent pas : elles sont, en quelque sorte, consolidées et leur densité faible ne variera pas (ou presque pas) en fonction des charges appliquées surtout si elles sont appliquées très lentement.

Il est à peu près certain que ce phénomène de liquéfaction s'est produit à Nice lors de l'accident du 16 octobre 1979. En effet, il est à peu près certain que dans les masses de terres qui sont parties, devaient se trouver des couches de sables lâches analogues à celles qui ont été identifiées ailleurs. Ces sables ont été évidemment soumis à de petites déformations, ce qui a forcément engendré leur liquéfaction, puis les grandes déformations. Les inclusions de sables liquéfiés ont évidemment formé un extraordinaire lubrifiant dans la masse dans le mouvement de glissement général.

\section{2}

\section{Liquéfaction des sables fins saturés sous l'effet d'un chargement cyclique}

Là encore, les travaux de B. Seed et de ses collaborateurs ont été, en leur temps, à l'avant-garde de la science. Ils sont cependant un peu plus anciens et, à ce titre, bien connus des spécialistes.

Ce phénomène est assez voisin du précédent. Lorsqu'un sable fin saturé est soumis à des sollicitations cycliques, la structure granulaire est capable de s'effondrer et de perdre toute résistance. Le nombre de cycles est d'autant plus grand que la densité relative du sable est élevée. Pour des densités faibles, la perte de résistance peut être obtenue par des sollicitations cycliques beaucoup plus petites que la sollicitation statique qui aurait entraîné la liquéfaction du sable. Le phé- 
nomène est lié à l'augmentation progressive, cycle après cycle, de la pression interstitielle.

Il ne semble pas que ce phénomène ait joué un rôle particulier à Nice lors de l'accident du 16 octobre 1979, puisque aucune sollicitation cyclique particulière (sismique, par exemple) n'a été identifiée.

\section{3}

\section{Liquéfaction des argiles hautement sensibles}

II s'agit d'un phénomène qui a été observé dans des argiles qui se sont déposées en conditions marines, puis qui ont été lessivées de leurs ions sodium par une percolation d'eau douce. Des formations de ce genre n'ont été identifiées que dans les pays nordiques, au Canada (Leda), en Suède et en Norvège (Rissa), et on les appelle quick clays. Ces argiles remaniées par une déformation perdent leur structure initiale. Comme leur teneur en eau naturelle est nettement supérieure à la limite de liquidité, une colline se transforme en un fleuve de boue. La résistance au cisaillement s'effondre dans le rapport de 1 à 20. De tels matériaux sont sensibles à une addition de chlorure de sodium, quí leur fait retrouver une part de la résistance initiale. La situation hydrogéologique du delta sous-marin du Var était favorable au lessivage de l'argile par l'eau douce, et on a pu comparer le cas de Nice à celui des argiles nordiques hautement sensibles à structure fragile. Indépendamment du fait que les conditions climatiques de dépôt dans la région de Nice n'ont rien de commun avec celles des argiles glaciaires, il était souhaitable d'en faire l'expérimentation. Les essais qui ont été faits n'ont pas permis de mettre en évidence une quelconque influence d'une addition de sel. Il ne semble donc pas que ce phénomène ait pu se produire dans les matériaux argilo-silteux du talus du delta du Var.

\section{4}

\section{Liquéfaction des argiles sensibles}

Bien qu'il ne s'agisse pas d'une liquéfaction au sens strict du mot, c'est-à-dire d'une perte quasi complète de la résistance, il est certain que les matériaux argilo-silteux du delta du Var doivent être considérés comme des matériaux sensibles, c'est-à-dire susceptibles de perdre notablement leur résistance lorsqu'on les remanie. Le radoucissement correspondant est important, et cet effet, allié à la présence des gaz inclus dans ces matériaux mous, rend leur prélèvement dit « intact », puis leur étude en laboratoire, particulièrement délicats. En effet, les gaz inclus se dilatent lorsqu'on amène les échantillons à la pression atmosphérique au moment du prélèvement, ce qui bouscule les grains et désorganise la structure déjà peu résistante de ces matériaux. La perte de résistance par le remaniement est suffisamment sensible pour qu'on lui ait attribué, avec une certaine vraisemblance, la faible cohésion trouvée immédiatement sous les remblais, les sols bousculés lors de la mise en place des remblais n'ayant pas récupéré leur résistance structurale initiale.

Le radoucissement de ces matériaux, allié à la lubrification par les couches de sables fins liquéfiés ou par l'eau de mer ambiante, a probablement contribué très largement à provoquer l'écoulement de toute la partie supérieure de la zone éboulée, c'est-à-dire celle qui portait la digue du nouveau port, en un temps très court (3 à $4 \mathrm{~min}$ ) pour un glissement de terrain, mais apparem- ment long pour un phénomène de liquéfaction complète du type de ceux qui ont été évoqués plus haut.

\section{Liquéfaction par augmentation de la pression interstitielle}

On dit parfois que l'augmentation de pression interstitielle peut amener un sol à la liquéfaction. On sait, par exemple, que la mise en place de remblais augmente la pression interstitielle du sol sous-jacent et aussi qu'elle peut se transmettre au loin par le canal de couches plus perméables ; ce phénomène pourrait entraîner la liquéfaction de l'argile sous le chargement, mais aussi, loin de la zone chargée.

Il s'agit d'une erreur de langage ; ce phénomène peut entrainer la rupture de l'argile mais non sa liquéfaction; on ne peut pas parler d'une pression interstitielle limite de liquéfaction, mais simplement d'une pression interstitielle critique telle que la stabilité locale (c'est-à-dire là où la pression interstitielle s'est propagée) soit en danger.

A ce titre, la pression interne au delta du Var, correspondant à une surpression hydrostatique de $5 \mathrm{~m}$ et peut-être davantage, après les fortes pluies d'octobre qui ont précédé la journée de l'accident, n'est pas un facteur de liquéfaction, mais simplement un facteur d'instabilité. Si une rupture se produit, la liquéfaction par radoucissement peut évidemment se produire, mais ultérieurement comme une conséquence de la déformation.

\section{Liquéfaction par effet d'avalanche}

L'avalanche sous-marine, comme l'avalanche de neige, est un phénomène apparenté à la mécanique des fluides. Il est important de savoir que ce fluide en écoulement est animé de mouvements turbulents, dont le sens de rotation est l'inverse de celui du roulement des blocs sur une pente lors d'une avalanche de blocs rocheux (Fig. 8).

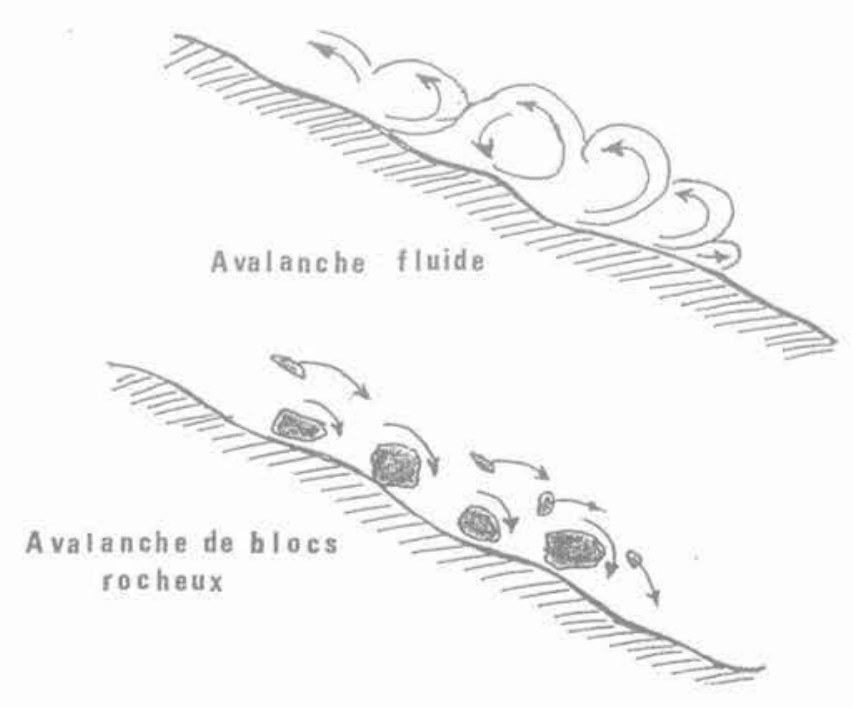

FIG. 8 Avalanche fluide et effondrement rocheux. Fluid avalanche and stones avalanche. 
Ces rouleaux de turbulence contribuent au maintien des terres en suspension dans le courant de densité, ce qui leur permet ainsi de s'écouler très au loin. Ces mouvements turbulents permettent sans doute, comme pour l'avalanche de neige, de mettre en mouvement toutes les terres instables disponibles sur les talus sur lesquels l'avalanche sous-marine déboule et de les maintenir en suspension ce qui est indispensable pour qu'un courant de densité puisse s'écouler.

\section{5}

\section{Remarques sur l'expertise}

Une expertise en cas de ruine mécanique doit se terminer par un calcul qui démontre que la résistance était inférieure à la sollicitation.

Par exemple voici trois fils concourants et une charge sur l'un des brins (Fig. 9). On calcule aisément les forces dans les deux autres brins ; si la résistance de l'un de ces deux brins est inférieure à la sollicitation, la cause de l'accident est trouvée. La justice peut déterminer ensuite qui est responsable ou comment se répartissent les responsabilités : le maître d'ouvrage qui n'a pas donné les véritables charges auxquelles le système sera soumis, le maitre d'œuvre qui s'est trompé dans le calcui des forces, l'entrepreneur qui a économisé sur les fils, le fournisseur qui n'a pas donné les bons fils, le contrôleur qui n'a pas bien fait les vérifications, etc.

Il ne faut pas demander au tribunal de se substituer aux experts et, en particulier, l'autorité de la chose jugée ne peut pas s'étendre à la partie technique de l'expertise. Sinon le soleil continuerait de tourner autour de la terre depuis le procès de Galilée.

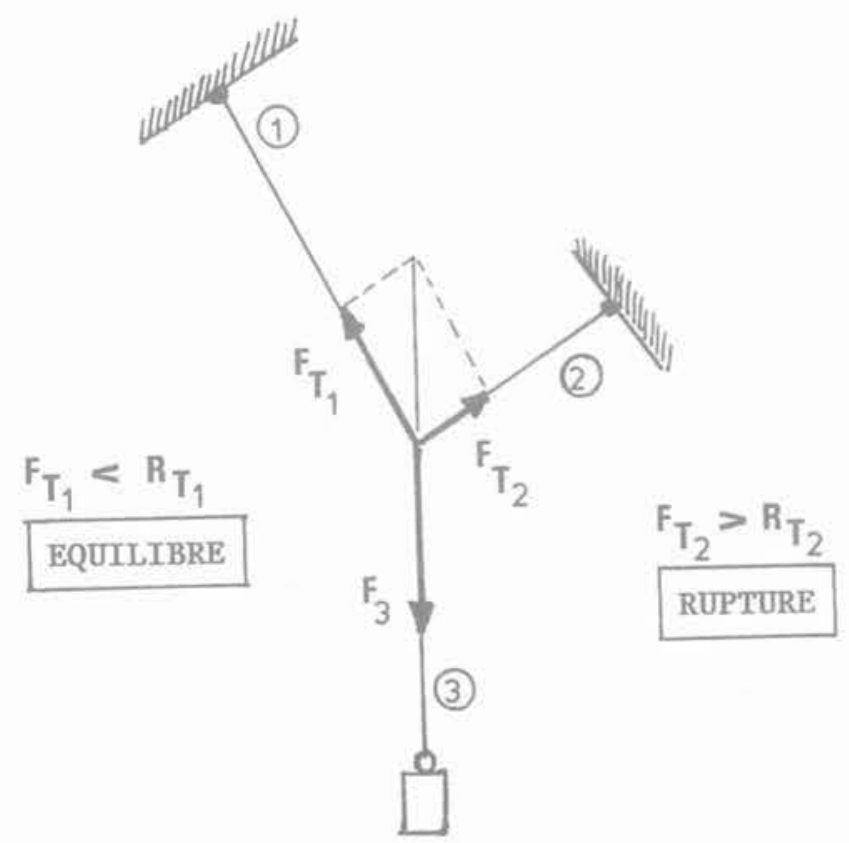

FIG.9 Équilibre ou rupture d'un système de trois fils.

$\mathrm{F}_{\mathrm{T}}=$ État-limite ultime de la force de tension du projet.

$\mathrm{R}_{\mathrm{T}}=$ État-limite ultime de la résistance à la traction du projet.

Equilibrium or failure of a three strings system.
Bien entendu, l'expertise commence par un examen et un diagnostic et c'est en ce sens que le travail de l'expert a souvent été assimilé à celui du médecin. Mais ce qui distingue ces deux activités, c'est la vérification numérique. Le travail de l'expert est souvent rendu difficile par le fait que tel petit défaut de la structure apparaît après la ruine comme une déchirure béante, et il faut imaginer la situation initiale où la géométrie était correcte.

Mais pour un glissement de terrain, lorsque le corps du délit a disparu, parce qu'un entrepreneur actif a terrassé toute la masse glissée, ou parce que celle-ci a disparu au fond des mers, il est extraordinairement difficile, voire impossible, de déterminer la résistance initiale. Nous avons dit plus haut que la résistance de ce qui est resté en place à Nice était plus grande que celle de la matière qui est partie : il s'agit d'un truisme qu'on peut répéter pour tout accident et il n'est pas étonnant que les calculs classiques faits dans l'hypothèse de la rupture circulaire, même avec des essais de laboratoire particulièrement délicats du fait des difficultés de prélèvement d'échantillons intacts signalés précédemment, et avec des essais in situ effectués dans des matériaux inhabituels, donnent des coefficients de sécurité compris entre 1,1 et 1,3. Ne connaissant ni les propriétés mécaniques, ni même les éléments géométriques qui ont caractérisé l'intérieur du talus avant son écroulement, on ne peut faire que des approximations grossieres qui n'ont pas valeur de preuves.

Dans ces conditions, on ne peut qu'imaginer des scénarios possibles, le plus possible de scénarios possibles, et les éliminer successivement chaque fois qu'une invraisemblance apparait. Mais que faire s'il reste plusieurs scénarios vraisemblables?

\section{6}

\section{Quelques scénarios possibles}

\section{1}

\section{Scénario proposé par la MIP}

Pour la MIP, le raz de marée observée en baie des Anges a été causé par un glissement de terrain d'origine non sismique. Par des considérations sur la vitesse de propagation des vagues et de la propagation des ondes sonores dans le sol, un certain nombre d'accidents possibles situés à plus de $3 \mathrm{~km}$ du chantier ont été écartés (élimination de scénarios); la MIP en a déduit que l'accident s'est passé au voisinage du nouveau port et en définitive que le glissement qui a entraîné le nouveau port est responsable du raz de marée :

" La MIP ne peut donner aucune cause précise à l'amorçage du glissement ayant affecté le port. „

« La MIP est arrivée à la conclusion qu'avant qu'il n'affecte le remblai du nouveau port, le glissement s'est manifesté sur le talus sous-marin pour s'étendre progressivement ensuite au plateau et aux remblais du port. Mais, en l'absence de toute information directe, on en est réduit aux hypothèses quant à la position et la cause du glissement. »)

Il s'agirait donc d'un glissement régressif, comme indiqué au $\$ 1$ dont la cause initiale est incertaine et ce n'est pas trahir l'esprit des conclusions de la MIP de dire que, du fait du caractère régressif de l'accident, la présence des remblais du nouveau port n'a en aucune manière contribué au déclanchement de l'accident (Fig. 10a), 


\section{Une variante sur ce scénario}

Le scénario retenu par la MIP présente une difficulté. Le premier mouvement qui a été observé au port a eu lieu à $13 \mathrm{~h} 57 \mathrm{~min} 30 \mathrm{~s}$. Les premiers mouvements observés à Saint-Laurent-du-Var ont eu lieu au plus tard à la même heure ; même si les vagues à la surface de l'eau peuvent aller très vite dans la baie des Anges, de $30 \mathrm{~m} / \mathrm{s}$ à $100 \mathrm{~m} / \mathrm{s}$ du fait de la profondeur, il faut au moins 30 s pour qu'un signal puisse aller du nouveau port jusqu'à Saint-Laurent-du-Var. Il semble donc que le début du raz de marée ait précédé le début du glissement du chantier du nouveau port qui du coup ne peut pas être considéré comme le facteur déclenchant du raz de marée. Il faut donc qu'un autre phénomène se soit produit avant la chute du port. On voit ici l'importance de la connaissance d'une chronologie exacte puisqu'une imprécision de l'ordre de la minute peut modifier complètement l'évaluation de la cause. Dans l'hypothèse cidessus, on peut imaginer un accident sous-marin au voisinage du nouveau port situé dans le canyon par où est passé l'éboulement. Le scénario est alors le suivant.

L'équilibre précaire du talus du delta du Var se dégrade progressivement dans la matinée du 16 octobre 1979. Vers $13 \mathrm{~h} 54$, un glissement s'amorce dans un thalweg instable du talus du delta du Var et se transforme en un grave accident en s'augmentant de toutes les terres instables du talus du Var; si l'on se souvient qu'il a fallu 3 à 4 min pour faire disparaître les 4 à 5 millions de mètres cubes correspondant à l'emprise du nouveau port, on admettra qu'il a bien fallu 2 ou 3 min pour faire effondrer les 3 à 4 millions de mètres cubes de la partie inférieure du glissement. Cet effondrement fait apparaitre une dépression de la surface de la mer qui provoque une baisse du plan d'eau du nouveau port et déstabilise celui-ci, cependant qu'un mouvement régressif se développe sur le plateau du talus du delta du Var. Le creux de vague ne se propage pas vers l'est, car il en est physiquement empêché par la présence de la digue le creux d'onde ne peut qu'irradier avec une très faible intensité vers l'aéroport en se diffractant à l'extrémité est de la dique. La partie basse du glissement repousse de l'eau devant elle et engendre un boursouflement de la surface de la mer qui part en précurseur positif vers Antibes et Port-Vauban. Le creux qui s'est produit se propage vers le reste de la côte et apparaît d'abord, notamment aux marégraphes, comme un premier reflux. C'est à peu près au moment où cette onde arrive au port de Saint-Laurent-du-Var que la dique du nouveau port disparaît à son tour et que la deuxième phase du glissement se produit, relançant une nouvelle vague devant elle qui complique le phénomène hydraulique. Ces mouvements fortement dissymétriques par rapport à la baie des Anges (et les prolongements sous-marins par les deux crêtes qui l'encadrent) mettent en résonance l'ensemble du bassin dans une oscillation qui perdra son énergie par radiation en quelques heures.

Les mouvements qui se sont produits autour du premier point d'effondrement ont engendré des distorsions dans les volumes d'alluvions situés près de cette zone de glissement. Tous les horizons de sables fins saturés lâches, identifiès par les reconnaissances à terre et en mer effectuées avec le piézocône, se sont presque instantanément liquéfiés. Ces couches, en perdant toute résistance, ont reporté plus haut (et plus bas) les efforts qu'elles supportaient, engendrant ainsi de nouvelles distorsions qui provoquaient de nouvelles liquéfactions. Les limons sensibles soumis à de grandes déformations se sont radoucis au point de ne plus offrir qu'une résistance médiocre. Tout s'est alors écoulé en passant entre les deux piliers restés en place, probablement consolidés depuis plus longtemps et situés de part et d'autre du chenal d'écoulement. Finalement, il est resté au niveau du nouveau port un fond faiblement incliné et sur le talus du delta du Var une pente d'érosion plus forte. Les matériaux remaniés de l'effondrement se sont d'abord écoulés dans le canyon du nouveau port, puis dans le canyon du Var, cependant que le mécanisme d'avalanche grossissait le courant de densité de tout ce qui était instable sur le chemin et transportait à plus de $100 \mathrm{~km}$ de là l'ensemble des débris (Fig. 10b).

\section{3}

\section{Scénario de B. Seed}

Pour B. Seed, toutes les indications horaires de l'arrivée des manifestations sur la côte sont trop imprécises pour qu'il soit possible d'en tirer des informations exactes : les observateurs n'ont pas tous vu le début du phénomène, que ce soit un creux d'onde ou un petit gonflement, voire ont cru voir la première vague quand c'était la seconde ou la troisième. En fait tous les témoignages pourraient s'accorder sur une perturbation venant du large, de très loin, $15 \mathrm{~km}$ peut-être, qui aurait été engendrée par l'effondrement de quelques cent millions de mètres cubes de sédiments. Les arrivées sur la côte ont été presque simultanées, éventuellement avec quelques petites différences du fait de la longueur des trajectoires et de la célérité des ondes de surface qui est variable car fonction de la profondeur de la mer.

Cette perturbation initiale a provoqué un raz de marée le long de la côte, d'Antibes â Beaulieu, avec un abaissement du niveau de l'eau de l'ordre de $2,50 \mathrm{~m}$. Au nouveau port, les remblais ont été déjaugés ce qui a créé une augmentation soudaine de la charge sur le sol du plateau du delta du Var et cela s'est traduit par une augmentation de $2 \%$ du déviateur des contraintes à l'endroit où est apparue la première rupture sur le talus du delta du Var. Ce fut suffisant pour que cette sollicitation statique rapide déclenche après un certain temps la liquéfaction dans les couches de sables lâches et cette première rupture a été suivie d'une évolution régressive qui a emporté le nouveau port (Fig. 10c).

Pour déterminer l'endroit où la première rupture s'est produite du fait de la baisse du plan d'eau, B. Seed fait un calcul par la méthode des éléments finis sur un modèle représentant le profil initial du talus du delta du Var. La sollicitation est apportée en tête par le poids propre et par l'effet du déjaugeage des remblais. On s'intéresse uniquement à ce qui se passe sur le profil restant du sol après l'accident (Fig. 11); il y a évidemment sur cette ligne un point où le déviateur de rupture est maximal et B. Seed dit que ce point est celui où s'est produit la liquéfaction initiale. Hasard ou intuition, ce point est voisin de celui où la MIP avait situé l'origine de l'accident régressif qui a emporté le nouveau port.

\section{Commentaires}

Dans les deux premiers scénarios cités ci-dessus, l'origine de l'accident initial reste obscure et c'est bien désagréable. Mais il faut dire que toutes les autres interprétations qui ont pu être proposées, y compris celle de B. Seed, présentent la même faiblesse, et ont besoin d'un deus ex machina pour le début de l'accident : le mystère reste entier pour savoir ce qui a pu déclencher les événements sous-marins ayant provoqué le raz de marée sur la côte. 


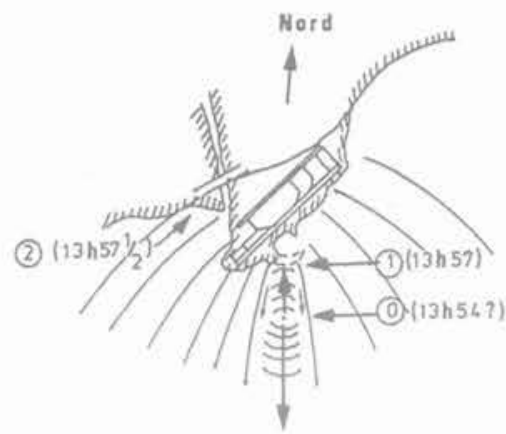

a)

La vague qui atteint Saint-Laurent-du-Var a $13 \mathrm{~h} .57 \mathrm{~min} 30$ s est déclenchée par le glissement du nouveau port quil commence 13 h 57

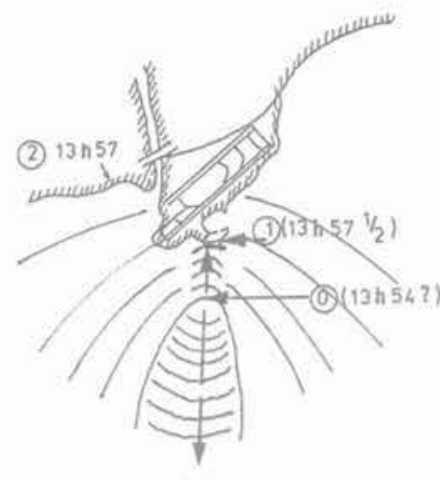

b)

La vague qui atteint Saint-Laurent-du-Var a 13 h 57 est déclenchée par un premier glisse. ment sous-marin qui commence à 13 h 54 (?) Le glissement régressif détruit le nouveau port à 13 h 57 min $30 \mathrm{~s}$

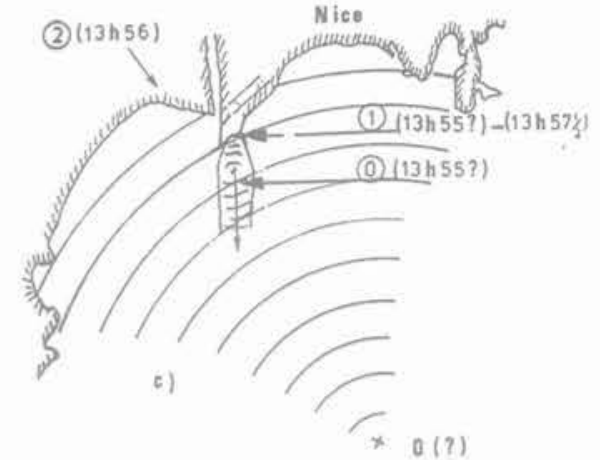

La vague qui atteint le nouveau port à 13 h 55 (?), puis la côte à 13 h 56 est déclenchée par un glissement sous-marin lointain qui provoque une rupture locale à 13 h 55 (?) puis la chute du port à $13 \mathrm{~h} 57$ 1/2.

FG. 10 Trois scénarios : (a) d'après la MIP; (b) variante de (a) ; (c) d'après B. Seed. Three scenarios : (a) adapted from MIP; (b) variant of $(a)$; $(c)$ adapted from B. Seed.

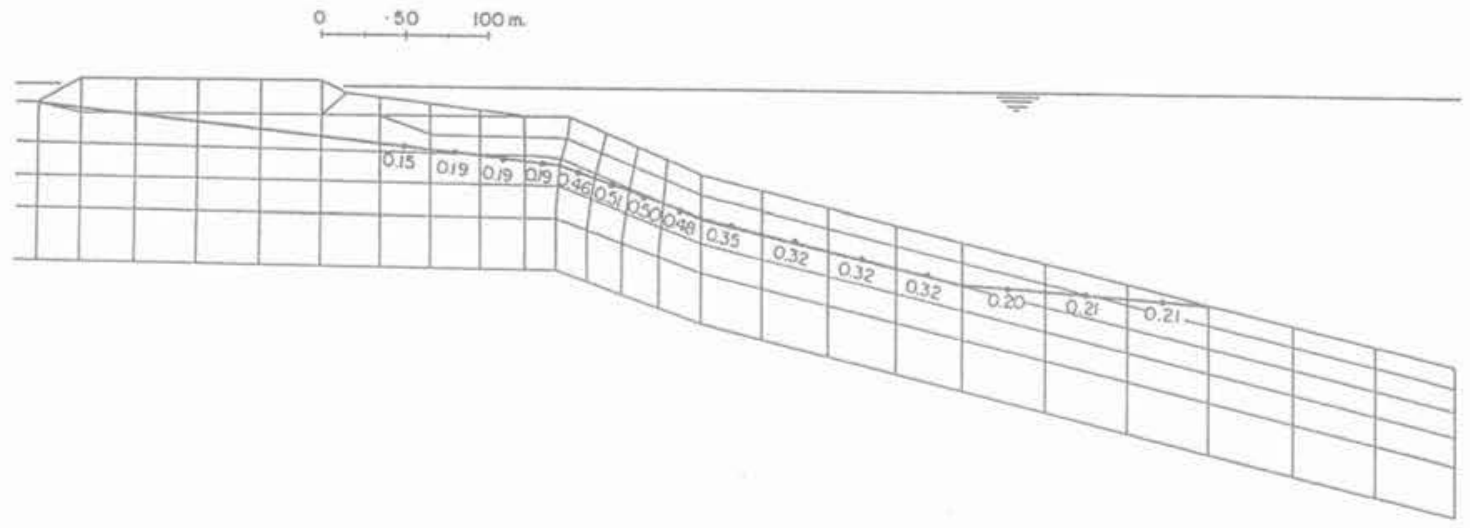

FIG. 11 Calcul de $\tau / \sigma$ par la méthode des éléments finis sur la surface restante après l'accident (d'après B. Seed).

Calculation of $\tau / \sigma$ by finite elements method on the remaining surface after the accident (from B. Seed).

Le mécanisme de la rupture par l'effet du temps, qui est appelé en rhéologie la fatigue statique, est un des plus difficiles à appréhender et aussi un de ceux pour lesquels la dispersion expérimentale est la plus grande. Voici un arbre mort qui reste debout. Après cinq ans, il s'effondre. Pourquoi cinq ans et pas six? La prévision de son altération est difficile. Dans le cas présent, il s'agit du pourrissement du bois dont on comprend la progression avec le temps. Mais, pour un assemblage de minéraux, c'est beaucoup moins clair. Pourtant, cela existe. Une carrière de craie à Clamart s'est effondrée, en faisant 21 victimes, en 1961 après trente ans d'abandon ; elle avait été estimée instable au moment de l'arrêt de l'exploitation par A. Mayer qui était alors ingénieur en chef des Mines au service des Carrières et qui a été ultérieurement président du CFMS : pendant vingtneuf ans, cette carrière a défié toute prévision : elle a donné raison à l'expert la trentième année. On ne peut donc pas écarter une rupture du talus sous-marin par " vieillissement $»$, ce mot étant pris au sens large, effet de l'érosion, de la fatigue statique, de l'artésianisme, etc. Tous les scénarios proposés sont donc vraisemblables ; il est regrettable qu' on ne puisse les confirmer par l'analyse mathématique de l'équilibre précaire du talus, mais en l'absence de témoignage et à cause de la disparition du corps du délit (c'est-à-dire les matériaux glissés), il est bien évident qu'aucun calcul n'est vraiment possible. Ne connaissant ni les propriétés mécaniques, ni même les éléments géométriques qui ont caractérisé l'intérieur du talus avant son écroulement, on ne peut faire que des approximations tout à fait insuffisantes, comme par exemple les hypothèses d'homogénéité et d'isotropie du sol pour le calcul par éléments finis. 


\section{Risque sismique du talus du delta du Var}

Il est instructif de rapprocher l'accident du 16 octobre 1979 de trois raz de marée historiques qui ont frappé Antibes, en 1564, en 1818 et en 1887, après des séismes ressentis à terre. En 1979, il n'y a pas eu de séisme avant l'accident, de sorte qu'on est obligé de faire appel au mécanisme de la fatigue statique. Avec des séismes et des matériaux reconnus comme liquéfiables dans les alluvions du Var, il est évident que la liquéfaction provoquant un glissement sous-marin du talus du delta du Var est une hypothèse géologique tout à fait satisfaisante pour expliquer les raz de marée historiques.

Mais, on peut dire encore que si un séisme avait eu lieu quelques jours avant le 16 octobre 1979, il est à peu près certain, dans l'état de précarité des talus, qu'il aurait déclenché à son tour un nouveau raz de marée historique. On peut même aller encore plus loin, en constatant que l'accident du nouveau port n'a purgé qu'un seul des sillons qui festonnent le talus du delta du Var, et qu'après tous les autres ne doivent pas être en meilleur état; on peut dire qu'un danger d'effondrement spontané des talus continue d'exister et qu'un séisme pourrait effectivement en être le facteur déclenchant. La présence reconnue au cours des travaux d'expertise, tant en mer qu'à terre, par des essais in situ au piézocône, de matériaux liquéfiables dûment identifiés, renforce cette hypothèse. En cas de séisme, la liquéfaction des sables lâches fins saturés en l'absence de travaux de confortation pourrait provoquer à nouveau un grave accident dans le talus du Var et même dans la plateforme aéroportuaire, car il n'y a aucune consolidation à attendre d'un sable, même lâche, sous contrainte statique.

Cette notion de risque sismique m'est apparue tellement évidente à la lecture des différents documents au cours de l'analyse des travaux d'expertises que j'ai interrogé sur ce sujet les trois principaux experts géotechniciens qui ont participé à l'analyse de l'accident à savoir, G. L'Hériteau, J. Goguel et B. Seed. Leurs réponses indépendantes ont été affirmatives pour tous trois et s'ils n'ont pas exprimé officiellement cet avis, c'est simplement parce que cette question ne faisait pas partie de leur mission. Mais il n'est guère besoin de forcer beaucoup les textes des experts pour mettre en évidence cette opinion. Ainsi la MIP dans son rapport publié du 15 juillet 1980 écrit : « Le risque d'un grand glissement pouvant résulter d'un séisme ne peut, lui, être écarté mais la probabilité d'un tel événement est très faible. »

Je possède d'ailleurs un manuscrit de G. L'Hériteau indiquant clairement que la MIP s'était rangée à l'avis de J. Goguel pour penser qu'un séisme entraînerait la rupture du talus naturel du delta du Var en de nombreux points.

Quant à B. Seed, dans son rapport final, s'il a repoussé, du fait de l'absence de phénomènes vibratoires liés par exemple à des manifestations sismiques, l'éventualité du déclenchement du phénomène de liquéfaction, donc du glissement, par un mécanisme de vibration cela prouve bien, a contrario, que dans son esprit, les vibrations d'un séisme pouvaient engendrer une rupture du talus. D'ailleurs, l'essentiel de l'ceuvre de Seed sur les phénomènes de liquéfaction des sables fins saturés sous l'effet des sollicitations cycliques a été consacré à ce risque.

\section{Conclusion}

L'analyse qui vient d'être présentée du sinistre survenu le 16 octobre 1979 à Nice montre la difficulté d'interpréter un accident de cette ampleur, Iorsqu'on ne dispose que de témoignages partiels et que le corps du glissement a disparu, rendant fragile l'interprétation géotechnique. Il n'en reste pas moins qu'après les analyses remarquables de la MIP, de la DDE et de B. Seed, quelques éléments sont suffisamment certains (la structure interne du talus du delta du Var, la précarité de l'équilibre global, la présence de matériaux liquéfiables, ...) pour qu'un scénario explicatif puisse être proposé. Ceux qui ont été présentés ici ne sont pas les seuls qui soient possibles. Ils paraissent cependant assez raisonnables ; ils présentent tous la même lacune, à savoir que le déclenchement initial reste un peu mystérieux, mais soulignent l'existence d'une instabilité sismique potentielle du talus du delta du Var.

\section{$\overline{\text { Bibliographie }}$}

[1] Crabatti - La dinamica della frana del Vaiönt, Giornale di Geologia, vol, XXXII, fasc. I, pp. $139-154,1964$.

[2] Edgers L,, Karisrud K, - Soil Flows Generated by Submarines Slides-Cases Studies and Consequence, Norges Geoteck- niske Institutt. Publication $\mathrm{n}^{\circ}$ 143, Osio, 1982

[3] Larsson R., Jansson M.- The landslide at Tuve Nov. 30, 1977, Rapport $\mathrm{n}^{\circ} 18$, Swedish Geotechnical Institute, 1982

[4] Marcello - La catastrophe du Vaiönt, $8 \bullet$ Congrès Internatio- nal des Grands Barrages, pp. 562-572, 1964.

[5] Mulder Th. Tissot J.-P., Cochonnat P. Bourillet J.-F. - Stabilité des pentes sous-marines dans la baie des Anges, Nice, France. Approche géotechnique, Revue française de géotechnique, $n^{\circ} 64$, pp. $21-30,1993$.
[6] Mulder Th. - La vitesse du courant de turbidité de 1979 à Nice, Apport de la modélisation. C.R. Ac. Sc. Tome $317 n^{\circ} 11$, 2 déc. 1993, p. 1449.

[7] Vidart D. - Tsunami, vague et. tempéte, Les défis du CEA, $n^{\circ} 18$, Paris, pp. 20-22, 1993. 